\title{
Pelvic Curve
}

National Cancer Institute

\section{Source}

National Cancer Institute. Pelvic Curve. NCI Thesaurus. Code C33289.

A normal anteroinferior concavity of the sacrum and coccyg eal vertebrae, extending from the lumbosacral junction to the apex of the coccyx. 